\title{
NUEVAS ADICIONES DE BIVALVOS A LA MALACOFAUNA MARINA PERUANA
}

NEW ADDITIONS OF BIVALVIA TO THE PERUVIAN MARINE MOLLUSKS

\author{
Carlos Paredes y Franz Cardoso*
}

\section{RESUMEN}

Cuatro especies de moluscos bivalvos marinos, Nuculana (Jupiteria) cuneata (Sowerby, 1833), Isognomon (Isognomón) janus Carpenter, 1857, Ostrea conchaphila Carpenter, 1857 y Mysella (Rochefortia) molinae Ramorino, 1968 se reportan por primera vez para el mar peruano. Se incluye datos sobre la distribución geográfica y el hábitat de cadia una de las especies.

Palabras claves: Moluscos bivalvos, nuevos registros, Perú.

\section{ABSTRACT}

Four species of marine bivalve mollusks, Nuculana (Jupiteria) cuneata (Sowerby, 1833), Isognomon (Isognomon) janus Carpenter, 1857, Ostrea conchaphila Carpenter, 1857 and Mysella (Rochefortia) molinae Ramorino, 1968 are reported for the first time from Peruvian sea. Data are included on the geographical distribution and habitat of each species.

Key words: Bivalve mollusks, new records, Perú.

\section{INTRODUCCIÓN}

En el transcurso de las investigaciones sobre la diversidad de los invertebrados del mar peruano, dentro del proyecto "El bentos y su interacción en el ecosistema marino somero", se ha venido realizando monitoreos en diversos lugares y biotopos del subsistema béntico, con el objetivo de elaborar un Catálogo de los Moluscos Marinos del Perú. Al respecto, Álamo y Valdivieso (1997) revisaron los reportes de moluscos bivalvos marinos del Perú, considerando los trabajos clásicos de Dall (1909) y Keen (1971), así como los de Vegas-Vélez (1968), Peña (1971) y Valdivieso (1984). Posteriormente, otros autores como Bernard (1983), Cruz y Jiménez (1994), Hendrickx y

*Laboratorio de Invertebrados Acuáticos, Facultad de Ciencias Biológicas, UNMSM, Apdo. 11-0058, Lima 11, Perú. E-mail: d190043@unmsm.edu.pe
Toledano (1994), y Goto y Poppe (1996) han actualizado la sistemática y distribución de los bivalvos marinos.

En el presente trabajo se reporta, por primera vez para el Perú, cuatro especies de bivalvos marinos, incluidas en las familias Nuculanidae, Isognomonidae, Ostreidae y Montacutidae.

\section{MATERIAL Y MÉTODOS}

Los muestreos en algunas localidades ( $\mathrm{Ba}-$ hía de Ancón y Bahía Independencia) de la costa peruana se realizaron mensualmente, y en otras en forma esporádica. Los ejemplares se recolectaron en la orilla rocosa y en algunos casos mediante una draga van Veen en la zona infralitoral. El material fue fijado con formol al 7\% neutralizado con bórax y conservado en alcohol etílico al $70 \%$, con excepción de las 
conchillas, que se mantienen en seco. En el laboratorio se hizo las mediciones y tomas fotográficas. Para la determinación taxonómica se utilizó la bibliografía disponible, y para el ordenamiento de los taxa supraespecíficos se siguió a Vaught (1989). Este material está depositado en la colección del Laboratorio de Invertebrados Acuáticos de la Facultad de Ciencias Biológicas, Universidad Nacional Mayor de San Marcos.

\section{RESULTADOS}

Subclase Protobranchia

\section{Orden NUCULOIDA}

Familia Nuculanidae

Nuculana (Jupiteria) cuneata (Sowerby, 1833), (Figura 1).

Nuculana (Saccella) cuneata, Ramorino, 1968: 189-191, lám. 1, figs. 3 y 8, lám. 4, fig.
3; Bernard, 1983: 12.

Concha pequeña, opaca, de color pajizo u oliva grisáceo; posteriormente alargada y rostrada; superficie ornamentada con crestas regulares concéntricas; charnela con 10 a 17 dientes anteriores y 8 a 15 posteriores, ligamento pequeño, negruzco y ubicado sobre un profundo condróforo trapezoidal. Longitud $10,0 \mathrm{~mm}$; altura $6,8 \mathrm{~mm}$.

Distribución geográfica: Entre Mejillones y Valparaíso, Chile (Ramorino, 1968; Bernard, 1983).

Nueva localidad: Bahía Independencia (14 $\left.15^{\prime} \mathrm{S}, 76^{\circ} 10^{\prime} \mathrm{W}\right)$, Pisco.

Hábitat: Fondo areno-fangoso, $54 \mathrm{~m}$ de profundidad.

Material examinado: 1 lote, 24 ejemplares, 30-08-86.

Observaciones: En general, las características

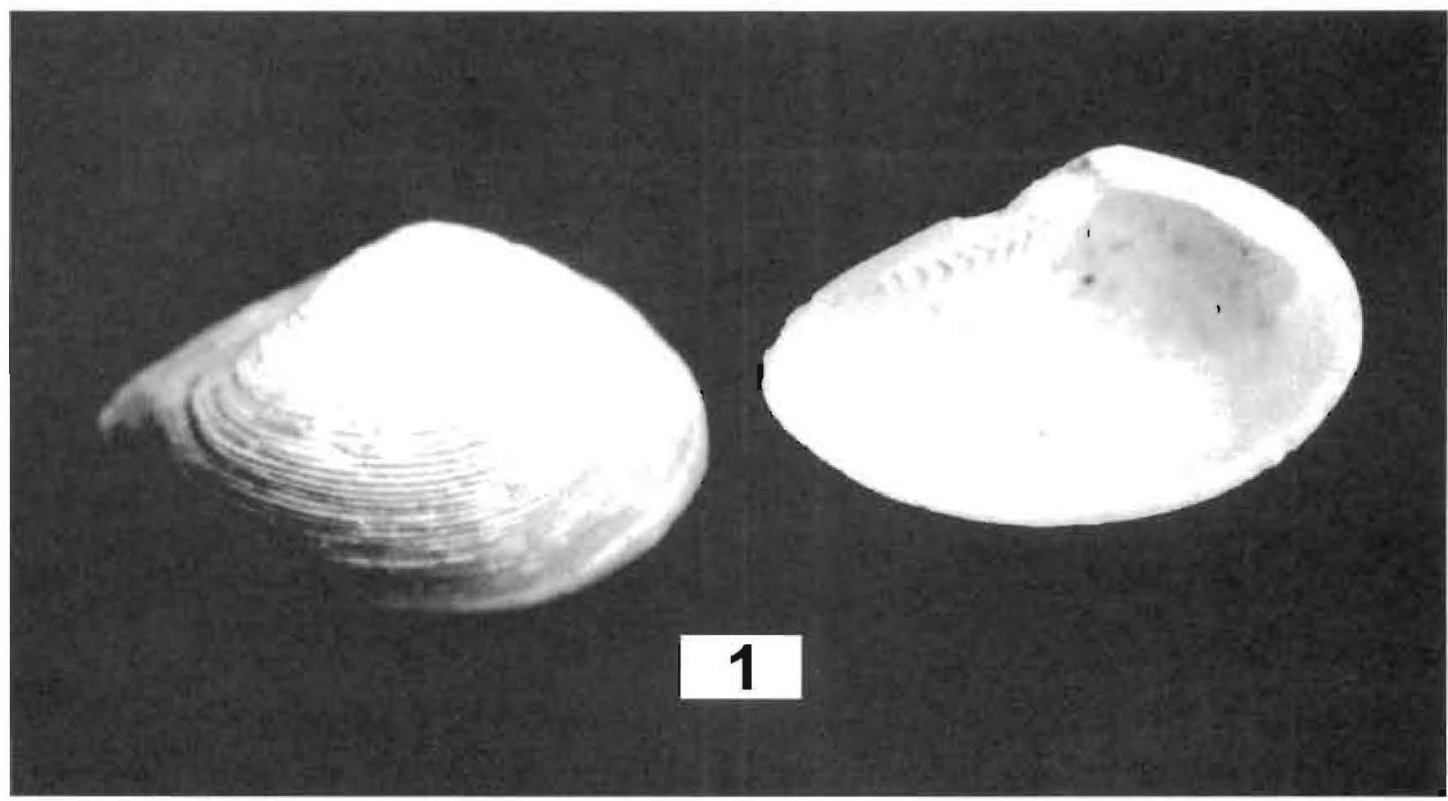

Figura 1. Nuculana (Jupiteria) cuneata, longitud $10,0 \mathrm{~mm}$ 


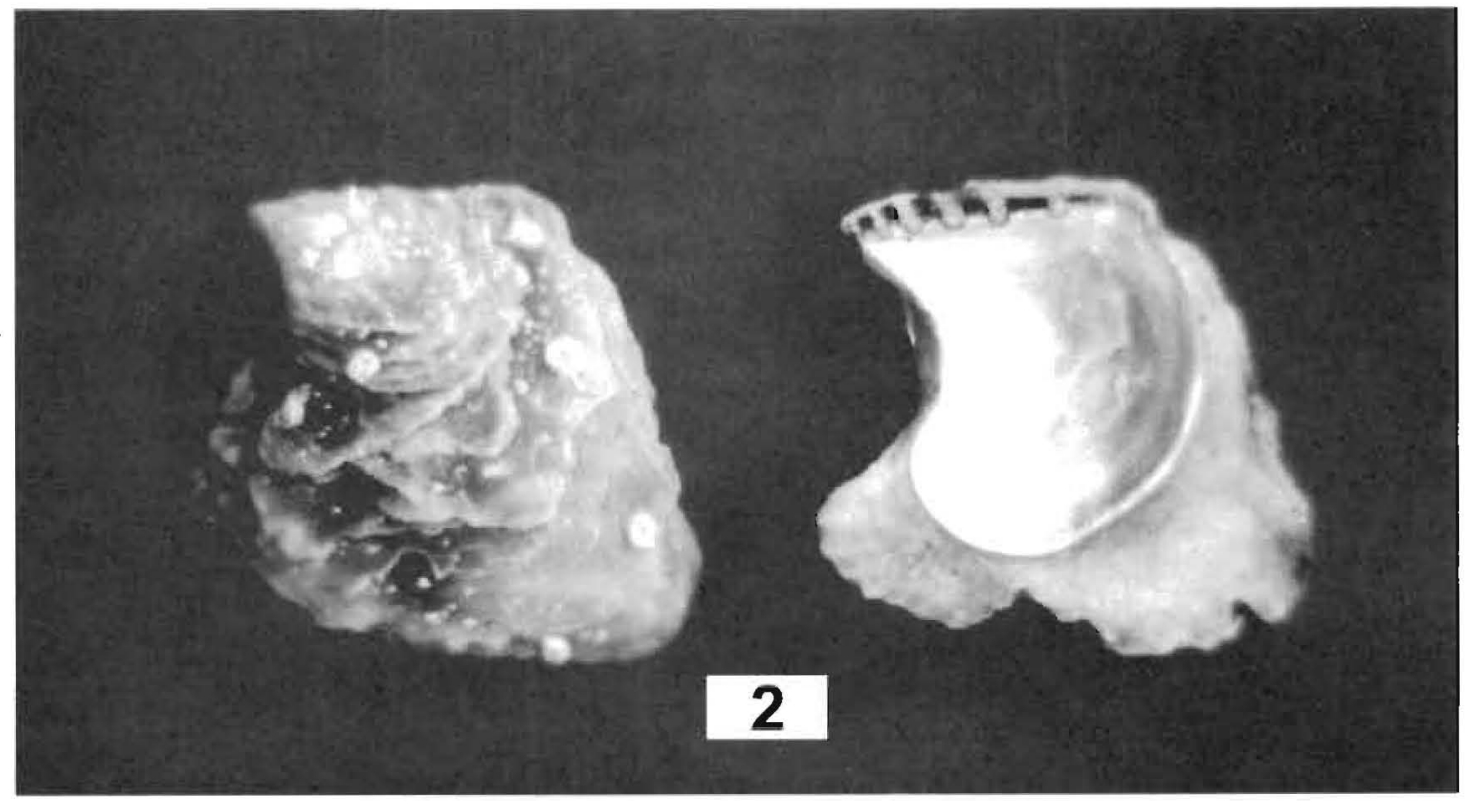

Figura 2. Isognomon (Isognomon) janus, longitud 20,0 mm

son similares a las dadas por Ramorino (op. cit.).

Subclase Pteriomorphia

Orden PTERIOIDA

Familia Isognomonidae

Isognomon (Isognomon) janus Carpenter, 1857, (Figura 2).

Isognomon janus Carpenter, 1857: 151; Goto y Poppe, 1996: 894; Keen, 1971: 79, fig. 163; Bernard, 1983: 22.

Conchilla de color pardo amarillento, más alta que larga, líneas de crecimiento que desarrollan formando escamas; escultura radial bien pronunciada en el margen ventral de la conchilla; la charnela presenta seis hendiduras o surcos ligamentales en cada valva. Longitud $20,0 \mathrm{~mm}$, altura $20,0 \mathrm{~mm}$.

Distribución geográfica: Baja California hasta Oaxaca, México (Keen, 1971).

Nueva localidad: Islas Lobos de Tierra.

Hábitat: Mediolitoral de la orilla rocosa, adheridos a rocas y fijado a otras conchas.
Material examinado: 1 lote, 3 ejemplares, 2807-97.

Observaciones: Esta especie es muy similar a I. recognitus (Mabille, 1895), la que se distribuye desde Baja California, Ecuador (Bernard, 1983) hasta Chile (Keen, op. cit.; Cruz y Jiménez, 1994). De acuerdo a la descripción dada por Keen (op. cit.), I. janus presenta una escultura radial bien pronunciada y sin lamelas concéntricas, y charnela con 7 a 9 surcos ligamentales, mientras 1 . recognitus una escultura lisa a escamosa, y charnela con 6 a 12 surcos ligamentales.

\section{Orden OSTREOIDA}

Familia Ostreidae

Ostrea conchaphila Carpenter, 1857 (Figura 3).

Ostrea conchaphila Carpenter, 1857: 161; Goto y Poppe, 1996: 900; Keen, 1971: 82, fig. 169; Bernard, 1983: 23.

Típicamente la concha es delgada, aproximadamente circular y chata; el exterior está listado radialmente de pardo oscuro, púrpura o tam- 
and Aquatic Sciences 61: 1-102.

Carcelles, A. R. y S. I. Williamson. 1951. Catálogo de los Moluscos marinos de la Provincia Magallánica. Revista del Instituto Nacional de Investigación de las Ciencias Naturales, Ciencias Zoológicas, 5: 225-383.

Carpenter, P. P. 1857. Catalogue of the Reigen collection of Mazatlan Mollusca in the British Museum. Warrington, England, Oberlin Press, 552 pp.

Cruz, R. A. y J. A. Jiménez. 1994. Moluscos asociados a las áreas de manglar de la Costa Pacífica de América Centra!: Guîa. Heredia, C. R.: EFUNA, $182 \mathrm{pp}$.

Dall, W. H. 1909. Report on a collection of shells from Perú, with a summary of the littoral marine Mollusca of the Peruvian Zoological Province. Proc. U. S. N. Mus., 37(1704): 147-294.

Goto, Y. y G. T. Poppe. 1996. A listing of living mollusca. Part II, Volume 2. L'Informatore Piceno, Ancona-Italy, pp. 525-1031.

Hendrickx, M. E. y A. Toledano. 1994. Catálogo de Moluscos. Colección de Referencia, Estación Mazatlán, ICML, UNAM. Comisión Nacional para el Conocimiento y Uso de la Biodiversidad e Inst. de Cienc. Mar y Limnol., UNAM, México D. F., 71 pp.

Keen, A. M. 1971. Sea shells of Tropical West
America.2nd. ed. Stanford Univ. Press, California, $1064 \mathrm{pp}$.

Mansur, M. C. y M. O. Da Silva. 1990. Morfología e microanatomía comparada de Bartlettia stefanensis (Moricand 1856) e Anodontitis tenebricosus (Lea 1834) (Bivalvia, Unionoida, Muteloidea). Amazoniana, 12(1): 147-166.

Peña, M. 1971. Zonas de distribución de los bivalvos marinos del Perú. An. Cient. Univ. Nac. Agraria, $9(1 / 2)$ :46-55.

Purchon, R. D. 1987. Classification and evolution of the Bivalvia: An analytical study. Phil. Trans. R. Soc. Lond., (B) 316:277-302.

Ramorino, L. 1968. Pelecypoda del fondo de la Bahía de Valparaíso. Rev. Biol. Mar., 13(3):175-285.

Valdivia, K.; R. Valdivia; P. Huamán y J. Zaldivar. 1995. Clave de identificación para géneros de bivaivos amazónicos (Mollusca: Unionoida) en el Perú. Biotempo, 2:97-103.

Valdivieso, V. 1984. Moluscos bivalvos del mar peruano. Boletín de Lima, 34:84-96.

Vaught, K.C. 1989. A classification of the living Mollusca. American Malacologist Inc., Florida, 189 pp.

Vegas-Velez, M. 1968. Revisión taxonómica y zoogeográfica de algunos gasterónodos y Iamelibranquios marinos del Perú. An. Cient. Univ. Nac. Agraria, 6(1/2):1-29. 